\title{
Hyperbaric Oxygen Therapy Alters Vascular Reactivity Independent of Elevations in ATP
}

\author{
Benjamin Hake ${ }^{1}$, Cole Waggener ${ }^{1}$, Benjamin Eovaldi ${ }^{2}$, Claude Zanetti $^{3}$ and Francis Sylvester ${ }^{1}$
}

Hyperbaric oxygen (HBO) is a pharmacologic therapy used treat a variety of medical conditions. The underlying mechanisms of HBOs effect on vascular reactivity are poorly understood. The purpose of this study was to record the acute effects of HBO on vascular reactivity and determine the potential role of ATP in mediating these effects. Porcine mesenteric arteries were dissected and mounted in isolated organ baths to record changes in tension in response to potassium chloride $(\mathrm{KCl}, 15-60 \mathrm{mM})$, phenylephrine (PHE, 10 $0^{-7}-10^{-4} \mathrm{M}$ ), and sodium nitroprusside (SNP, 10 $0^{-7}-10^{-4} \mathrm{M}$ ) following a 2-hour exposure to HBO (1.75 ATA). HBO augmented responses to $\mathrm{KCl}$ and PHE compared to control arteries exposed to room air or nitrogen at 1.75 ATA as well as to room air at $1 \mathrm{~atm}$. When compared to the 1 ATA room air control, KCl-induced constriction was significantly increased for the HBO exposure. Treatment with HBO also augmented vascular responses to PHE and SNP relative to nitrogen, but not ambient air. We hypothesized that HBO increased ATP production in vascular smooth muscle leading to enhanced vascular reactivity. Consequently, ATP levels were measured in mesenteric arteries but no significant differences in ATP levels were observed regardless of hyperbaric treatment. Direct measurements of ATPs' effects on porcine vasculature with and without hyperbaric treatment resulted in no significant findings. These results suggest that HBO alters vascular reactivity independent of elevations in ATP.

Keywords: Hyperbaric oxygen; vascular reactivity; artery

\section{Introduction}

Ischemic reperfusion injury (IRI) is quickly becoming a leading problem in today's hospitals. As the average age in the United States population increases, the chances of IRI also increase due to the higher associated cardiovascular risks. Hypertension, obesity, hyperlipidemia, and diabetes all contribute to higher levels of morbidity and mortality in the clinical setting. IRI is the complex phenomenon that occurs following treatment which restores blood flow to the initial site of injury. ${ }^{26,30}$ Clinically there is already damage incurred by the initial ischemic event, which can be catastrophic. It is then compounded by further injury that occurs when blood flow is reintroduced. This is particularly destructive in the mesentery because ischemia with subsequent reperfusion can lead to acute respiratory distress syndrome. ${ }^{20,30}$

The lack of oxygen and nutrients during the initial ischemic event decreases the production of ATP. ${ }^{9,}{ }^{13}$ Lack of ATP causes a decrease in intracellular $\mathrm{pH}$ due to lactic acid accumulation. Insufficient ATP will also impair the sodiumpotassium ATPase pump, which in turn alters cellular membrane potential via a loss of potassium and gain of sodium. Additionally, the distribution of intracellular ions is altered with an elevation of intracellular calcium being observed. ${ }^{6}$ The change in ion concentrations leads to many deleterious consequences. The cell begins to swell, eventually leading to its death. Further, an increase in reactive oxygen species (ROS), due to the elevated levels of intracellular calcium, leads to oxidative stress and cell death. ${ }^{27,35}$

Although the ischemia is the primary source of injury, reperfusion poses an equal, if not greater, clinical problem. During the period of reperfusion, there is an exacerbated and accelerated injury to the previously hypoxic tissues. A burst in ROS generation causes damage in tissue surrounding the ischemic event that was previously unaffected. ${ }^{29}$ The rise in ROS may be in part from the reintroduced oxygen after the restoration of blood flow. Ischemia may also cause a loss of normal antioxidant defense mechanisms. ${ }^{23}, 31$ Without these, there is further damage to tissues that would otherwise function normally. Corresponding with the reintroduction of blood is an influx of leukocytes and plasma proteins which coincides with activation of the complement system. ${ }^{18}$ The subsequent inflammation results in new tissue damage.

The vasculature then performs a significant role in ischemia and reperfusion. Decrease in oxygen to vessels affects the endothelium by altering production of soluble mediators that act on vessel tone. $7,9,16$ By normally controlling homeostatic vasodilation and constriction the integrity of the vasculature is maintained. Consequently, the protective barrier function of the vascular endothelium is decreased by the deteriorating condition of the cells. ${ }^{22}$ An increase in growth factors is also associated with the lack of oxygen. ${ }^{25}$ During reperfusion, the endothelial cells initiate expression of adhesion molecules for leukocytes and neutrophils which leads to further damage of the tissue. 5,36

Interestingly, the location of the initial ischemic event can affect the progression of reperfusion syndrome. In particular, if the ischemic event occurs in intestinal regions of the body, an even more threatening progression occurs. In the mesentery, manifestations of both the ischemia and reperfusion injury lead to breakdown of the vasculature and intestinal mucosa. ${ }^{10,34}$ The resulting consequence is the escape of the intestinal flora. Once systemic, the bacteria can colonize the lungs and start a pathologic inflammatory response that leads to acute respiratory distress syndrome, a potentially life threatening disorder.

Hyperbaric oxygen (HBO) therapy has been shown to be an effective treatment option with disorders related to IRI. ${ }^{3,17}$, ${ }^{19} \mathrm{HBO}$ is a treatment in which the patient breathes $100 \%$ oxygen at pressures greater than atmospheric. HBO treatment is done within a range of pressures from 1.00 to 3.00 atmosphere absolute (ATA). At pressure levels of 3.00 ATA 
or above, neurotoxicity is observed. Clinically, a wide variety of applications are currently in use. HBO therapy is used to treat a variety of ailments, including decompression sickness, thermal burns, exceptional blood loss, crush injuries, compromised skin grafts, and carbon monoxide poisoning. ${ }^{8,39}$ Currently, the majority of literature involving HBO therapy and IRI has been focused on treatments of myocardial infarction and cerebral vascular accident. At present, HBO treatment is thought to work by increasing the partial pressures of oxygen in tissue, thereby making more oxygen available. ${ }^{14}$ HBO therapy has not only been shown to aid in vascularization in ischemic tissue but directly enhance fibroblast replication and collagen synthesis in ischemia. ${ }^{26}$ Some evidence suggest HBO stimulates blood cell progenitors, which aid in the recovery of damaged tissue, possibly utilizing a nitric oxide dependent pathway. ${ }^{37}$

There is presently a lack of substantial understanding of the effects of HBO on mesenteric arteries. Thus this study investigated the effects of hyperbaric treatment on mesenteric arteries in an attempt to increase our understanding of this increasingly utilized clinical treatment. Specifically, we examined the direct effects of $\mathrm{HBO}$ on vascular reactivity and ATP levels in porcine mesenteric arteries. We hypothesize that HBO treatment causes an increase in ATP production, thus enhancing vascular reactivity. Increased vascular reactivity could then confer some protection against reperfusion injury.

\section{Results}

\section{Optimal Tension}

In vivo, arteries have a continual amount of tension in their walls which can also be described as tone. As such, changes in tension within the arterial wall are key determinants of blood pressure. To be able to compare functionality of each arterial ring, the optimal tension in vitro for porcine mesenteric arteries was assessed. To determine this optimal passive tension, we placed the arterial rings in organ-tissue baths and subjected them to doses of potassium chloride $(\mathrm{KCl})$. Changes in tension were recorded at different passive tensions to see where the greatest change in tension would occur. The results of this study suggest that there were no significant difference in $\mathrm{KCl}$-induced vasoconstriction among the passive tensions in porcine mesenteric arteries. The results were plotted as a function of their passive tensions at both doses of $\mathrm{KCl}$ then analyzed for significance by ANOVA. The largest difference was seen at 17 grams of passive tension, as arterial tension increased 23.03 and 30.96 $\mathrm{g}$ in response to 30 and $60 \mathrm{mM} \mathrm{KCl}$. The next largest difference was observed at 7 grams of passive tension, 26.47 $\mathrm{g}$ when $60 \mathrm{mM} \mathrm{KCl}$ was administered. The weakest responses were observed at 3, 15, and 25 grams of passive tension. There was no significant difference found between weight and outer diameter measurements.

\section{Vascular Reactivity}

Once optimal passive tension was decided, the artery's vascular reactivity, i.e. its ability to change its diameter in response to a stimulus, was assessed in response to the various gas/pressure treatments. Arterial rings were placed in an organ-tissue bath and vasoconstriction followed by dilation was induced experimentally to determine their reactivity. The vascular reactivity for each arterial ring was defined as the change in tension from the initial passive tension set by the experimenter to the new recorded tension observed after administration of the stimulus. The vascular reactivity for arterial rings was compared among treatments. The results of this experiment show that vascular reactivity was significantly increased in HBO $(31.35 \pm 3.48 \mathrm{~g})$ when compared to the ambient air control $(19.81 \pm 2.06 \mathrm{~g})$ in response to $60 \mathrm{mM} \mathrm{KCl}$ (figure 1). Interestingly, the hyperbaric nitrogen treatment $(24.02 \pm 2.69 \mathrm{~g})$ performed better than ambient air control in response to $60 \mathrm{mM} \mathrm{KCl}$. Treatment with $\mathrm{HBO}$ augmented vascular responses to phenylephrine (PHE) relative to nitrogen but not ambient air (figure 2). At their largest differences $\left(10^{-5} \mathrm{M}\right), \mathrm{HBO}$ had a reactivity of $30.47 \pm 3.12 \mathrm{~g}$ as compared to hyperbaric nitrogen at $15.66 \pm 1.83 \mathrm{~g}$. The hyperbaric room air $(22.51 \pm 3.94 \mathrm{~g})$ and ambient room air $(23.18 \pm 3.19 \mathrm{~g})$ were not significantly different and similar in mean reactivity. Regression analysis showed a significant difference in mean changes in tension for sodium nitroprusside (SNP)-induced dilation for all other treatments compared to $\mathrm{HBO}$ (figure 3). For example at a concentration of $10^{-5} \mathrm{M}(\mathrm{SNP})$, HBO $(-23.66 \pm 3.19 \mathrm{~g})$ had the greatest response when compared to all other treatments. Nitrogen ($11.03 \pm 1.36 \mathrm{~g}$ ) was the least responsive while hyperbaric room air $(-15.46 \pm 2.6 \mathrm{~g})$ was less responsive than ambient room air $(-18.14 \pm 2.87 \mathrm{~g})$. Each arterial ring was weighed and outer diameter was measured for subsequent comparison to ensure that the observed changes in vascular reactivity were due to the different gas/pressure treatments and not differences in arterial ring size. There were no significant observed differences in outer diameter or weight among treatments (ANOVA). The outer diameter measurements of HBO, hyperbaric nitrogen, hyperbaric room air and ambient room air were $4.37 \pm 0.28 \mathrm{~mm}, 4.82 \pm 0.28 \mathrm{~mm}, 4.32 \pm 0.17 \mathrm{~mm}$ and $4.05 \pm 0.21 \mathrm{~mm}$ respectively. The weight measurements of HBO, hyperbaric nitrogen, hyperbaric room air and ambient room air were $0.030 \pm .002 \mathrm{~g}, 0.030 \pm .002 \mathrm{~g}, 0.029 \pm .002 \mathrm{~g}$ and $0.027 \pm .001 \mathrm{~g}$ respectively. Results were stated as mean \pm SEM.

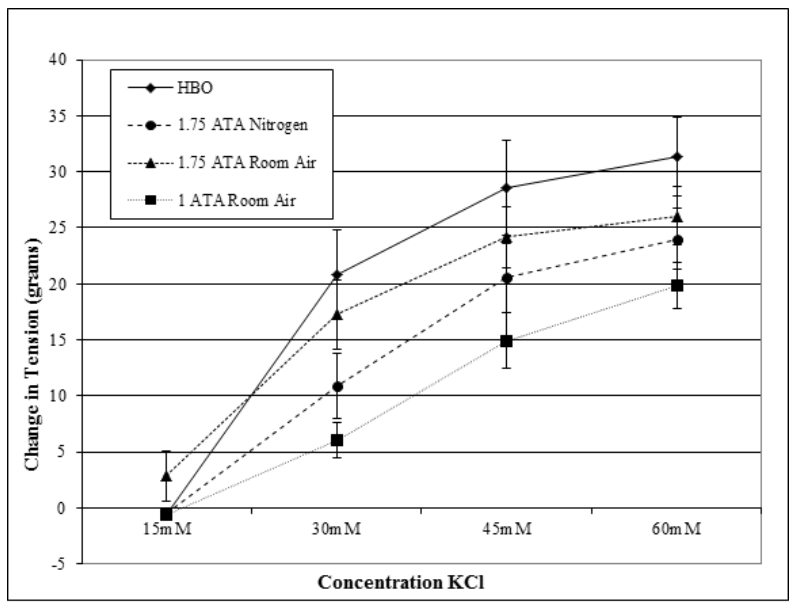

Figure 1: The effects of cumulative increases of $\mathrm{KCl}$ on arterial tension following 2 hour exposure to one of various hyperbaric conditions. Results are expressed as mean \pm SEM. * Response significantly different from ambient air at 1 ATA $(\mathrm{n}=12, \mathrm{p}<0.05$, ANOVA $)$. 


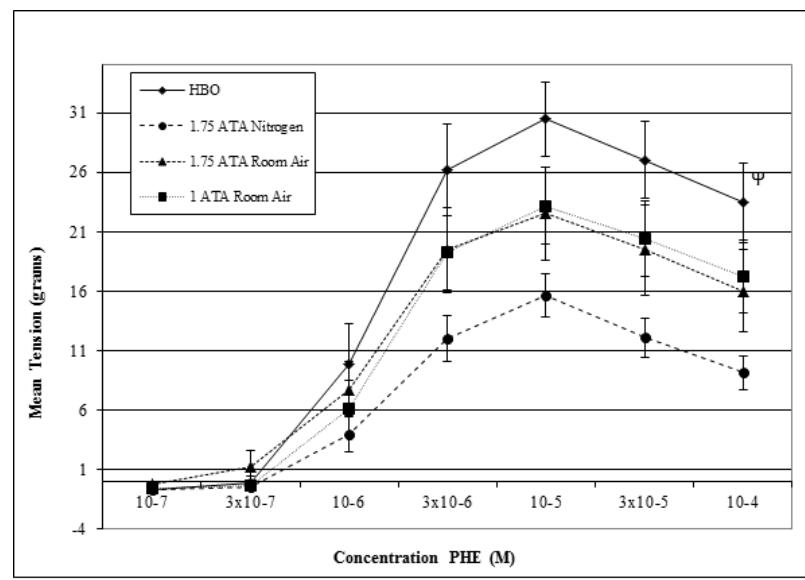

Figure 2: The effects of PHE on vasoconstriction of porcine arteries following 2 hour exposure to one of various hyperbaric conditions. The changes in arterial tension are differences measured in grams in response to increasing concentrations of PHE. Results are expressed as mean \pm SEM. ${ }^{\psi}$ Response significantly different from $100 \%$ nitrogen $(\mathrm{n}=12$ $\mathrm{p}<0.05$, regression analysis).

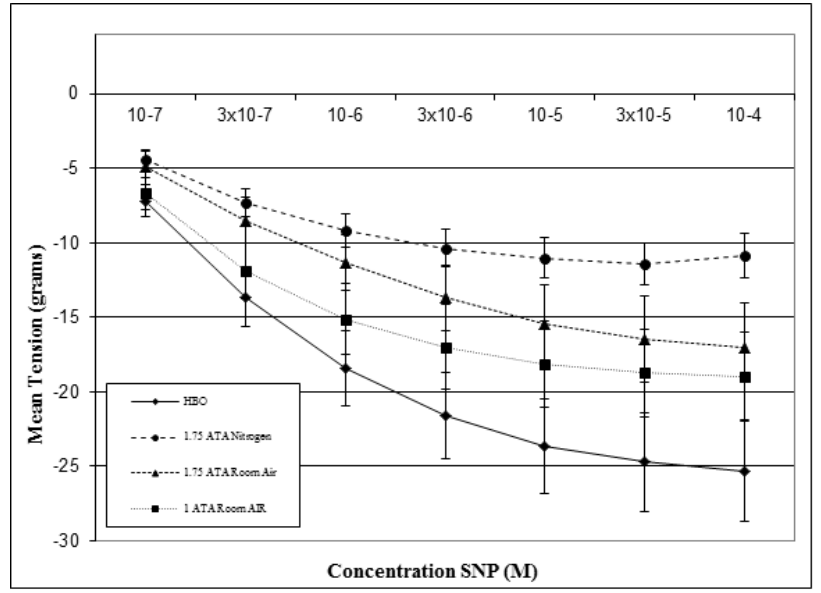

Figure 3: The effects of SNP on vasodilation of porcine arteries following preconstriction with PHE. Treatments were 2 hour exposures to one of various hyperbaric conditions. The changes in arterial tension are differences measured in grams in response to increasing concentrations of SNP. Results are expressed as mean \pm SEM. ₹ Response significantly different from all other treatments ( $n=12 \mathrm{p}<0.05$, ANOVA).

\section{ATP Analysis}

The previous experiments suggest that HBO increases porcine mesentery vascular reactivity. Given the ability of $\mathrm{HBO}$ to increase oxygen to the tissue, it could be possible that this increased oxygen availability causes increased ATP production. Increased ATP could account for the increases in reactivity observed. However, when ATP was measured following HBO treatment, it was found that ATP levels were not significantly increased in mesenteric arteries. HBO treatment $(0.2506 \pm 0.2010 \mathrm{nmol})$ did show the largest average ATP concentration per 1 microgram of protein when compared to all other treatments (figure 4). Hyperbaric nitrogen $(0.1141 \pm 0.2705 \mathrm{nmol})$ was the next largest average ATP concentration when compared to ambient air and hyperbaric room air showing the lowest average concentration. After consideration, it was determined that $100 \%$ oxygen at 1.0 ATA would make another suitable control and was therefore added to the ATP assay for comparison. The $100 \%$ oxygen at 1 ATA had a similar mean ATP concentration when compared to all other treatments and was therefore not significantly different. All protein concentration analyses were within the standard curve (data not shown). This demonstrated that each arterial ring contained approximately the same amount of protein.

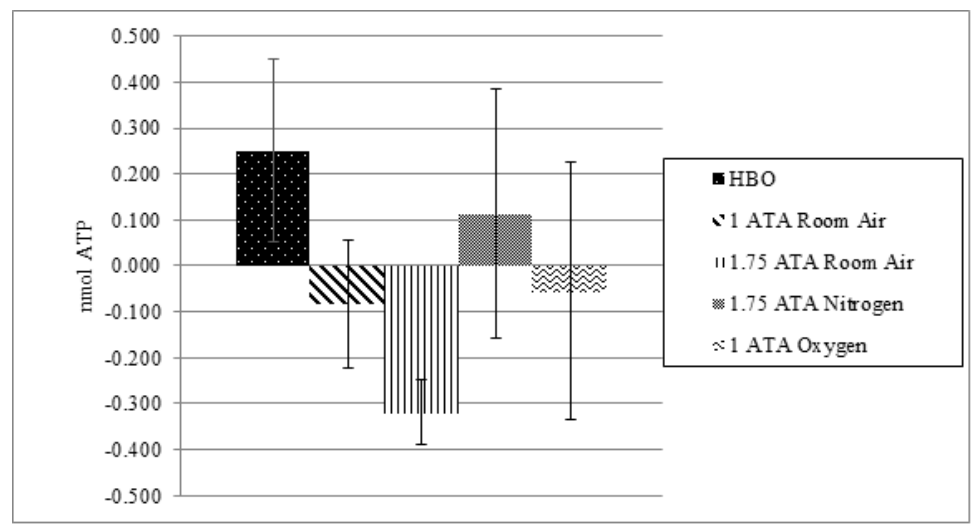

Figure 4: Comparison of ATP concentrations and pressure/gas treatments. Bars represent average nmol ATP per 1 microgram of protein from porcine mestenteric arteries following 2 hour exposure to one pressure/gas treatment. Colorometric concentration was measured at $570 \mathrm{~nm}$ with quantities being in the nanomolar range. Results are expressed as mean \pm SEM $(n=6)$. No significant difference was found among treatments (ANOVA).

\section{Direct effect of ATP on Vascular Rings}

Vascular rings were pretreated with a single $30 \mathrm{mM}$ dose of $\mathrm{KCl}$ to induce vasoconstriction. After the response to $\mathrm{KCl}$ plateaued, ATP or vehicle was added in increasing concentrations. As shown in Figure 5, the mean change in force for both ATP and vehicle, although increased, was not significant. The total mean change in force after the 20 minute period was less than two grams. This experiment was repeated without preconstruction with $\mathrm{KCl}$ and, once again, there was no significant response to exogenous ATP (data not shown). Vascular rings were exposed to a two-hour gas/pressure treatment, with the $100 \%$ oxygen treatment representing the HBO therapy, prior to the administration of the ATP or vehicle. As shown in figure 6 , all four treatments resulted in a decrease in the mean change in force which indicates vasodilation is occurring. However, the total mean change in force after the 20 minute period for all treatments is less than 1.5 grams with no observed significant difference among treatments. 


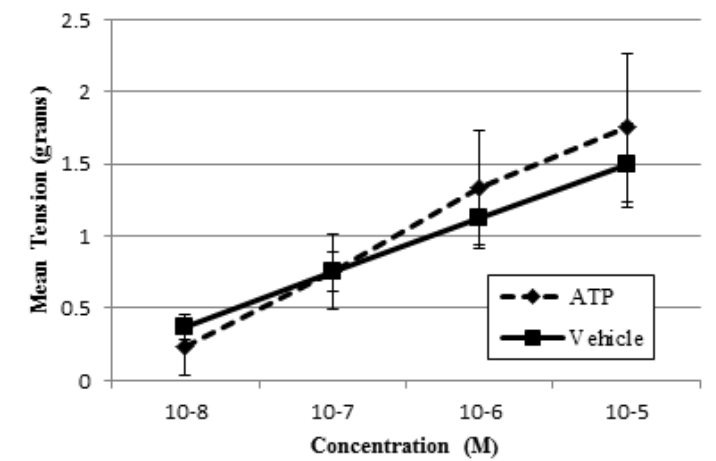

Figure 5: Vascular effect of ATP or vehicle on porcine arteries preconstricted with $30 \mathrm{mM} \mathrm{KCl}$. Values are expressed as mean \pm SEM $(n=5-9)$. No significant difference was found among treatments (ANOVA).

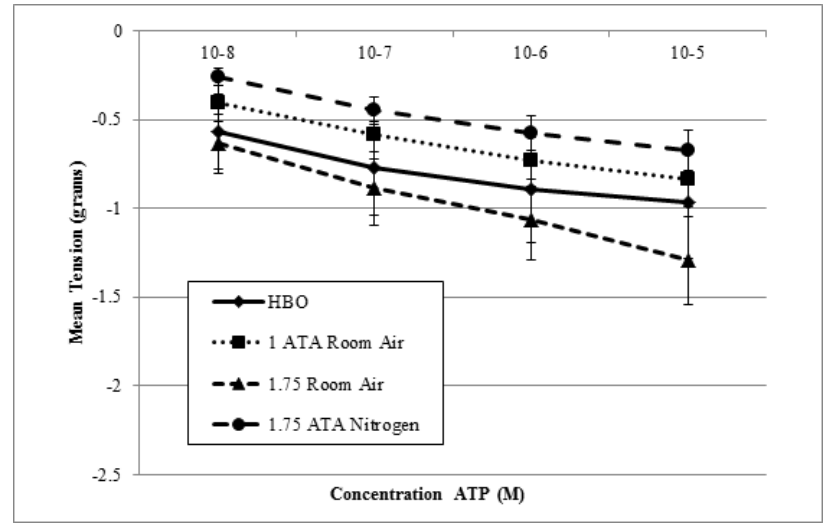

Figure 6: Vascular effect of ATP or vehicle on porcine arteries after one of four two-hour gas/pressure treatments. Values are expressed as mean \pm SEM $(n=7-8)$. No significant difference was found among treatments (ANOVA).

\section{Discussion}

This study demonstrated that HBO therapy improved the vascular reactivity of mesenteric arteries in response to $\mathrm{KCl}$, PHE, and SNP. However, the hypothesis that improved vascular reactivity is mediated by changes in ATP concentration in the mesenteric arteries was not supported by this study. The ATP concentrations of the mesenteric arteries did not significantly increase under exposure to $\mathrm{HBO}$ conditions (Figure 4).

Prior to the conduction of the vascular reactivity studies, an attempt was made to experimentally determine the optimal tension of mesenteric arteries studied in vitro. In this study there was no optimal tension observed. The length-tension relationship of muscle is an important consideration in the study of muscle contraction in the laboratory. The lengthtension relationship of vascular smooth muscle is dynamic. As observed in our study, arterial smooth muscle was able to produce forceful contractions at multiple passive tensions ranging from 1 to 25 grams. This same relationship is found in a study by Bednarek et al. (2011) utilizing arterial smooth muscle. ${ }^{2}$ The length-tension relationship was dynamic in rat femoral arteries and exhibited no clear optimal passive tension.

An artery's functional ability is measured by its capacity to constrict and dilate upon exposure to pharmacological and mechanical stimuli. $\mathrm{KCl}$ and PHE were used as pharmacological stimuli capable of inducing vasoconstriction. SNP was used as a vasodilator, pharmacological stimulus. Findings from other studies involving HBO therapy and its effects on vascular reactivity are scarce and conflicting. Although, HBO therapy's beneficial clinical effects are well recognized, little is known about HBO's direct effects on the vasculature. In one study, HBO treatment of rat aortic rings caused vasoconstriction independent of exogenous stimuli and decreased maximal constriction due to norepinephrine. ${ }^{17}$ In contrast, Imperatore et al. demonstrated that HBO therapy increased contraction to norepinephrine and endothelin-1 and dilation to acetylcholine when compared to the control group. ${ }^{19}$ There is scarce literature demonstrating the effects of $\mathrm{HBO}$ on $\mathrm{KCl}$-induced vasoconstriction in any arterial network. However, $\mathrm{KCl}$ is widely used in arterial ring studies as a potent vasoconstrictor. As shown in this study, exposure to $\mathrm{KCl}$ caused vasoconstriction of mesenteric arteries and this response was enhanced when arteries were given $\mathrm{HBO}$ therapy (figure 1). PHE is another commonly used vasoconstrictor in vascular reactivity studies.

PHE, a known vasoconstrictor, caused dose dependent increases in the force of contraction of arteries in the control and HBO groups. The vasoconstrictive effects of PHE were augmented by $\mathrm{HBO}$ exposure (figure 2). The general rational for use of $\mathrm{HBO}$ is that it increases the partial pressure and availability of oxygen to tissues and may ultimately lead to an increase in ATP production. ${ }^{11}$ Oxygen plays a vital role in ATP production. An increase in both oxygen's partial pressure and concentration could allow for increased oxygen utilization in vascular smooth muscle. Therefore, an increase in oxygen could confer an increase in ATP production. Increased cellular ATP levels would have advantageous effects for both vasoconstriction and dilation. Most notably, ATP plays a role in cross bridge cycling during excitation contraction coupling. The pump used to create a calcium gradient across the sarcoplasm in a smooth muscle cell is also an ATPase pump. Additionally, ATP serves as a modulator of vascular tone via ATP-sensitive potassium channels. ${ }^{33,40}$ Given ATP's significant role in smooth muscle regulation, alterations in ATP concentration were examined in attempt to elucidate a mechanism of action for $\mathrm{HBO}$ treatment. However, this study demonstrated no differences in ATP concentrations among treatment groups suggesting that ATP is not responsible for the observed alterations in vascular reactivity. A possible explanation for this occurrence is that during ATP determination, experimental controls did not accurately mimic in vivo conditions. For instance, ATP determination was not conducted at normal physiological temperature. Since oxygen is involved in multiple cellular processes such as respiration, signaling and regulation, it is quite possible that increased oxygen causes other unforeseen physiological reactions.

Based on the results of this study, simply increasing ATP concentration does not alter vascular reactivity. Future work should be directed towards investigating other possible mechanisms. Alterations in vascular tone may be caused by 
modulation of smooth muscle, the endothelium, or a combination thereof. Recently, oxygen levels have been implicated in regulating ATP-sensitive potassium channels, L-type $\mathrm{Ca}^{2+}$ channels and CYP450-4A enzymes, all of which regulate vascular responses. ${ }^{27}$ This is in support of a previous study that shows oxygen tension as a significant regulator of vascular reactivity. ${ }^{12}$ Oxygen tension has also been shown to alter endothelial cell respiration. ${ }^{21}$ Additionally, increased oxygen pressure has been shown to increase both nitric oxide synthesis and mitochondrial peroxide production. ${ }^{1,26}$ These can both have effects on vascular tone. Further, the endothelium has been shown to sense oxygen levels and react by releasing autacoids which control vascular reactivity. ${ }^{32}$

In summary, although ATP may not be responsible for the increased vascular reactivity, there remains a need to better understand the underlying mechanism associated with HBO therapy. HBO therapy has beneficial clinical applications for diabetic wound healing, organ transplant and ischemic reperfusion syndrome. It is therefore, a significant area of research to pursue in hopes of finding better treatments to various pathologies.

\section{Experimental Procedures}

\section{Animals \& Reagents}

All specimens were obtained from De Vries Meats Inc. of Coopersville, Michigan. Animals were procured from local farms, with inclusion of both female and castrated males, approximately 5 months old. A portion of fresh porcine mesentery often containing segments of duodenum and pancreas was placed on ice for transportation following exsanguination and evisceration.

\section{Dissection}

In the laboratory, arteries were dissected from the mesenteric tissue based on size and accessibility. Arteries chosen were approximately three to five millimeters in outer diameter and were at least ten millimeters in length. The specific branch from the porcine mesenteric network was unknown but believed to be either the right gastroepiploic artery or pancreaticduodenal artery due to relative size and position. Dissected arteries were cleaned of adherent connective tissue and either used immediately or placed in HEPES buffer solution for overnight storage at approximately $4^{\circ} \mathrm{C}$. HEPES buffer solution was composed of $10 \mathrm{mM}$ HEPES, $151 \mathrm{mM} \mathrm{NaCl}, 4.7 \mathrm{mM} \mathrm{KCl}, 2 \mathrm{mM} \mathrm{CaCl}_{2}, 1.2 \mathrm{mM}$ $\mathrm{MgCl}_{2}$, and $7.8 \mathrm{mM}$ glucose, with the $\mathrm{pH}$ adjusted to 7.4 . Cleaned arteries, either freshly dissected or following overnight storage, were placed in Krebs-Henseleit buffer solution and rings of approximately three to five millimeter in length were cut and left in solution. Krebs-Henseleit buffer solution had a composition of $118 \mathrm{mM} \mathrm{NaCl}, 4.7 \mathrm{mM} \mathrm{KCl}$, $1.2 \mathrm{mM} \mathrm{MgSO}_{4}, 1.25 \mathrm{mM} \mathrm{CaCl}_{2}, 1.2 \mathrm{mM} \mathrm{KH}_{2} \mathrm{PO}_{4}, 25 \mathrm{mM}$ $\mathrm{NaHCO}_{3}$, and $11 \mathrm{mM}$ glucose. All reagents were from SigmaAldrich (St. Louis, MO).

\section{Optimal Tension}

To obtain more reliable data for vascular reactivity experiments, determination of optimal tension was necessary. There is currently no definitive data on an optimal tension for porcine mesenteric arteries. Dissected arterial rings were mounted in individual baths on two steel hooks for isometric force measurement. Force transducers were attached to each hook to measure changes in tension. Each transducer was calibrated to 0 grams and 40 grams using standard masses. Measurements were recorded using Lab Scribe 2 software from iWorx Systems (Dover, NH). Each isolated organ bath contained $25 \mathrm{~mL}$ of freshly made Krebs-Henseleit buffer solution with temperature maintained at $37{ }^{\circ} \mathrm{C}$. $95 \% \mathrm{O}_{2}$ and $5 \% \mathrm{CO}_{2}$ gas was bubbled into each bath. Arterial rings were stretched at randomly assigned passive tensions $(1-25$ grams at each odd increment) for a 1-hour equilibration period. Arterial rings were randomly assigned using Excel by Microsoft (Redmond, WA). Each bath was drained, refilled, and tensions readjusted to respective magnitudes every ten minutes. Following equilibration, arteries were treated with a cumulative dose of $\mathrm{KCl}, 30$ then $60 \mathrm{mM}$ to assess reactivity. Changes in tension were recorded continuously at each dose. At the completion of the procedure, each artery was removed from the bath, weighed and outer diameter measurements were taken. Arteries were then discarded. Data were plotted using Excel. An ANOVA statistical analysis was completed with Sigma Stats by Systat Software (San Jose, CA).

\section{Hyperbaric Treatment}

Dissected arterial rings were placed in a Petri dish of Krebs-Henseleit buffer solution and positioned in a continuous-flow hyperbaric chamber. The hyperbaric chamber was custom built metal cylinder (approximately 29 $\mathrm{cm}$ inner-diameter with a depth of $8 \mathrm{~cm}$ ). For treatment, arterial rings were subjected to a 2-hour hyperbaric treatment of either $100 \%$ oxygen at 1.75 ATA, $100 \%$ nitrogen at 1.75 ATA, or room air at 1.00 or 1.75 ATA.

\section{Vascular Reactivity}

Immediately following hyperbaric treatment, arterial rings were mounted in isolated organ baths containing $25 \mathrm{~mL}$ of Krebs-Henseleit buffer solution. Baths were maintained at $37^{\circ} \mathrm{C}$ with $95 \% \mathrm{O}_{2}$ and $5 \% \mathrm{CO}_{2}$ gas bubbled into each bath. The arterial rings were stretched to a passive tension of 7 grams and subjected to a 1-hour equilibration period in KrebsHenseleit solution that was changed and tension readjusted to 7 grams every ten minutes. Following equilibration, arteries were treated with cumulative doses of $\mathrm{KCl}(15,30,40,60$ $\mathrm{mM})$ to induce vasoconstriction and assess viability. Arteries exhibiting an increase in tension of at least 5 grams from passive tension were used in further experimentation. Viable arteries were rinsed 3 times with fresh Krebs-Henseleit solution over a 15 minute period before being treated with PHE to induce vasoconstriction and SNP to induce vasodilation. Arteries were then treated with increasing concentrations of PHE $\left(10^{-7}, 3 \times 10^{-7}, 10^{-6}, 3 \times 10^{-6}, 10^{-5}, 3 \times 10^{-5}\right.$, $\left.10^{-4} \mathrm{M}\right)$ followed by increasing concentrations of SNP $\left(10^{-7}\right.$, $\left.3 \times 10^{-7}, 10^{-6}, 3 \times 10^{-6}, 10^{-5}, 3 \times 10^{-5}, 10^{-4} \mathrm{M}\right)$. Changes in tension were recorded continuously at each dose. The cumulative changes in tension represent each artery's ability to function properly. At the completion of the procedure, each artery was removed from the bath, weighed and outer diameter measurements were taken as a way to normalize the results for comparison. Results were plotted on Excel; statistical analysis was completed with SPSS software by IBM SPSS Statistics (Armonk, NY). An ANOVA was conducted for the weight and outer diameter measurements. Data were analyzed using Sigma Stats and plotted using Excel. PHE was 
purchased from Spectrum Chemicals (Gardena, CA). SNP was from Sigma-Aldrich (St. Louis, MO).

\section{ATP Analysis}

\section{Sample Preparation}

Arteries were dissected as previously described, except an individual $15 \mathrm{~mm}$ arterial section was cut into rings of three to five millimeters and all rings were kept and placed in Krebs-Henseleit buffer solution. Outer diameter measurements were taken for each ring prior to being cut into rings. Arteries were then subjected to hyperbaric treatment as previously described. Following treatment, arteries were removed from the Krebs-Henseleit solution, placed in $2.0 \mathrm{~mL}$ cryo.s snap tubes (Greiner Bio-one, Frickenhausen, Germany) and immediately frozen in liquid nitrogen. Frozen samples were stored at $-80^{\circ} \mathrm{C}$ for later processing. Frozen arteries were homogenized cold, using a mortar and pestle in $1.5 \mathrm{~mL}$ of $2 \mathrm{M} \mathrm{HClO}_{4}$. The perchloric acid was purchased from Sigma-Aldrich (St Louis, MO). Approximately $1.25 \mathrm{~mL}$ of homogenate was transferred to new $1.5 \mathrm{~mL}$ economy micro snap tubes (VWR, Chicago, IL). Samples were centrifuged at $0^{\circ} \mathrm{C}$ for 3 minutes at $12,000 \mathrm{rpm}$. The supernatant was removed and refrozen in liquid nitrogen for further analysis.

\section{Protein Concentration Assay}

Protein concentration determination was carried out as per the instructions by Thermo Scientific for the Coomassie Plus (Bradford) Assay Kit (item \#23236). Bovine serum albumin (Thermo Scientific, Rockford, IL) was used to generate a standard curve. For preparation of the standards, 2 $\mathrm{M} \mathrm{HClO}_{4}$ was used as the diluent. Samples were thawed but kept on ice during preparation. All other reagents were allowed to come to room temperature. When preparing and appropriating samples or standards into respective microplate wells, care was taken to briefly vortex each for approximately 10 seconds before allocution. $10 \mu \mathrm{L}$ of sample and standard were placed in each well. All samples and standards were analyzed in triplicate. The plate was then placed on a plate rotator for 10 minutes to mix and aid in reduction of air bubbles formed in wells. Remaining air bubbles were burst using a sterile pipette tip. Plates were then mixed for 30 seconds immediately prior to plate reading at $595 \mathrm{~nm}$ (BioRad, iMark microplate reader, Hercules, CA). Results were plotted and analyzed in Excel.

\section{ATP Concentration Assay}

ATP concentration determination was carried out as per the instructions by Bio Vision (San Francisco, CA) for the ATP Colorimetric/ Fluorometric Assay Kit (item \#K354-100). Samples were thawed and kept on ice during preparation. When preparing and appropriating samples or standards into respective microplate wells, care was taken to briefly vortex each for approximately 10 seconds before allocution. For each well the sample was transferred and adjusted to $10 \mu \mathrm{L}$ as necessary with $2 \mathrm{M} \mathrm{HClO}_{4}$. All samples and standards were analyzed in triplicate. Microplates were covered and placed on a plate rotator for 30 minutes to mix and reduce air bubbles. Any remaining air bubbles were burst using a sterile pipette tip. Measurements were taken at $570 \mathrm{~nm}$ (BioTek Synergy H1 Hybrid Multi-Mode Microplate Reader,
Winooski, VT) with results being plotted and analyzed in Excel.

\section{Direct effect of ATP on Vascular Rings}

Vascular rings were dissected out as previously described. The vascular rings were then placed in the organ bath for 45 minutes to one hour. During this period, the tension on the force transducer was adjusted to seven grams and the Krebs-Henseleit solution was replaced every ten minutes. Following equilibration, the vascular rings were administered increasing concentrations of $15 \mathrm{mM}, 30 \mathrm{mM}, 45$ $\mathrm{mM}$, and $60 \mathrm{mM} \mathrm{KCl}$, at five minute intervals, followed by $1 \times 10^{-7} \mathrm{M}, 1 \times 10^{-6} \mathrm{M}, 1 \times 10^{-5} \mathrm{M}$, and $1 \times 10^{-4} \mathrm{M}$ SNP also waiting five minutes between doses. The vessels were equilibrated and tested for viability using $\mathrm{KCl}$ to verify vasoconstriction and SNP to verify vasodilation. The organ baths were emptied and the vascular rings were washed three times in KrebsHenseleit solution every five minutes. The organ baths were refilled with $25 \mathrm{~mL}$ of Krebs-Henseleit solution and the vascular rings were exposed to increasing concentrations of $1 \times 10^{-8} \mathrm{M}, 1 \times 10^{-7} \mathrm{M}, 1 \times 10^{-6} \mathrm{M}$, and $1 \times 10^{-5} \mathrm{M}$ ATP (Enzo Life Science, Farmingdale, NY) or vehicle alone (deionized water), waiting five minutes between doses. If the vessel was constricted with $30 \mathrm{mM} \mathrm{KCl}$ before the addition of ATP, the organ baths were washed three times with Krebs-Henseleit solution for five minutes each and refilled with $25 \mathrm{~mL}$ of Krebs-Henseleit solution. The vessels were then given a single dose of $30 \mathrm{M} \mathrm{KCl}$ for fifteen minutes to preconstrict the vessels. The preconstricted vessels were treated with $1 \times 10^{-8} \mathrm{M}, 1 \times 10^{-7} \mathrm{M}, 1 \times 10^{-6} \mathrm{M}$, and $1 \times 10^{-5} \mathrm{M}$ ATP, waiting five minutes between doses. At the conclusion of the experiment, the vascular rings were dried then weighed, and the outer diameter was measured using a caliper.

Other data was collected using a similar procedure to that previously described but with notable exceptions. Vascular rings were placed in Krebs-Henseleit solution, or, if doing two runs in one day, the vessels of the second run were placed in the long-term storage buffer, HEPES with an adjusted $\mathrm{pH}$ of 7.4. Prior to the vascular studies rings were placed in the Krebs-Henseleit solution then into the hyperbaric chamber under one of the four gas/pressure treatments $\left(100 \% \mathrm{O}_{2}\right.$ at $26 \mathrm{psi}, 100 \% \mathrm{~N}_{2}$ at $26 \mathrm{psi}$, room air at 26 psi, and room air at atmospheric pressure) for two hours. The vessels stored in HEPES solution were switched into the Krebs-Henseleit solution before being placed in the hyperbaric chamber. Following hyperbaric treatment, the vessels were placed on the force transducers in the organ bath as previously described. The vessels were equilibrated and tested for viability using $\mathrm{KCl}$ and SNP. Viable vascular rings were exposed to ATP as in the above procedure; however no preconstriction of the vascular rings using $\mathrm{KCl}$ was performed. At the conclusion of the experiment, the vascular rings were dried then weighed, and the outer diameter was measured using a caliper.

\section{Acknowledgments}

We thank DeVries Meats, Inc. of Coopersville, MI for their generous donations of porcine mesentery necessary for this study. We also thank Matt Figlewicz for his help in preforming some of the ring studies. Finally, thanks to the Statistical Consulting Center at Grand Valley State University for their help with some of the statistical analysis. 


\section{References}

1) Baynosa RC, Naig AL, Murphy PS, Fang XH, Stephenson LL, Khiabani KT, Wang WZ, Zamboni WA The effect of hyperbaric oxygen on nitric oxide synthase activity and expression in ischemia-reperfusion injury. Journal of Surgical Research. 2013; 183(1): 355-61.

2) Bednarek ML, Speich JE, Miner AS, Ratz PH. Active tension adaptation at a shortened arterial muscle length: inhibition by sytochalasin-D. American Journal of Physiology Heart and Circulation Physiology. 2011; 300(4): H1166-H1173.

3) Bertoletto PR, Fagundes DJ, Simones MJ, Oshima CTF, Montero EF, Simoes RS, Fagundes AT. Effects of hyperbaric oxygen therapy on rat intestinal mucosa apoptosis caused by ischemia-reperfusion injury. Microsurgery. 2007; 27(4): 224-227.

4) Buettner, M., Wolkenhauer, D. Hyperbaric oxygen therapy in the treatment of open fractures and crush injuries. Emergency Medicine Clinics of North America. 2007; 25(1): 177-88

5) Carden DL, Granger DN. Pathophysiology of ischaemiareperfusion injury. Journal of Pathology. 2000; 190(3): 255-266.

6) Carrozza JP, Bentivegna LA, Williams CP, Kuntz RE, Grossman W, Morgan JP. Decreased myofilament responsiveness in myocardial stunning follows transient calcium overload during ischemia and reperfusion. Circulation Research. 1992; 71(6): 1334-1340.

7) Cipolla MJ, Bullinger LV. Reactivity of brain parenchymal arterioles after ischemia and reperfusion. Microcirculation. 2008; 15(6): 495-501.

8) Davis J. Hyperbaric oxygen therapy. Intensive Care Medicine.1989; 4: 55-57.

9) Eltzschig HK, Collard CD. Vascular ischaemia and reperfusion injury. British Medical Bulletin. 2004; 70: 71-86.

10) Fink MP, Delude RL. Epithelial barrier dysfunction: a unifying theme to explain the pathogenesis of multiple organ dysfunction at the cellular level. Critical Care Clinics. 2005; 21(2): 117-196.

11) Fisher BR, Palkovic S, Holling M, Wolfer J, Wassman $\mathrm{H}$. Rationale of hyperbaric oxygen in cerebral vascular insult. Current Vascular Pharmacology. 2010; 8(1): 3543.

12) Franco-Obregón A, Ureña J, López-Barneo J. Oxygensensitive calcium channels in vascular smooth muscle and their possible role in hypoxic arterial relaxation. Proceedings of the National Academy of Sciences of the United States of America: Physiology. 1994; 92(10): 4715-4719.

13) Frenguelli BG, Wigmore G, Llaudet E, Dale N. Temporal and mechanistic dissociation of ATP and adenosine release during ischaemia in the mammalian hippocampus. Journal of Neurochemistry. 2007; 101(5): $1400-1413$.

14) Gesell LB. (2008) Hyperbaric oxygen therapy indications. The hyperbaric oxygen therapy committee report. Durham, NC: Undersea and Hyperbaric Medical Society.
15) Groot H, Rauen U. Ischemia-reperfusion injury: processes in pathogenic networks: A Review. Transplant Proceedings. 2007; 39(2): 481-484.

16) Hein TW, Ren Y, Potts LB, Yuan Z, Kuo E, Rosa RH, Kuo L. Acute retinal ischemia inhibits endotheliumdependent nitric oxide-mediated dilation of retinal arterioles via enhanced superoxide production. Investigative Ophthalmology and Visual Science. 2012; 53(1): 30-36.

17) Hink J, Thom SR, Simonsen U, Rubin I, Jansen E. Vascular reactivity and endothelial NOS activity in rat thoracic aorta during and after hyperbaric oxygen exposure. American Journal of Physiology Heart Circulation Physiology. 2006; 291(4): 1988-1998.

18) Hughes SF, Hendricks BD, Edwards DR, Bastawrous SS, Roberts GE, Middleton JF. Mild episodes of tourniquet-induced forearm ischaemia-reperfusion injury results in leukocyte activation and changes in inflammatory and coagulation markers. Journal of Inflammation. 2007; 4: 12.

19) Imperatore $F$, Cuzzocrea $S$, Luongo $C$, Liguori $G$, Scafuro A, Angelis A. Hyperbaric oxygen therapy prevents vascular derangement during zymosan-induced multiple-organ-failure syndrome. Intensive Care Med. 2004; 30(6): 1175-1181.

20) James IA, Chen CL, Huang G, Zhang HY, Velten M, Besner GE. HB-EGF protects the lungs after intestinal ischemia/reperfusion injury. Journal of Surgical Research. 2010; 163(1): 86-95.

21) Jones CI, Han Z, Presley T, Varadharaj S, Zweier JL, Ilangovan G, Alevriadou BR. Endothelial cell respiration is affected by the oxygen tension during shear exposure: role of mitochondrial peroxynitrite. American Journal of Physiology Cell Physiology. 2008; 295(1): C180-C191.

22) Kong SE, Blennerhassett LR, Heel KA, McCauley RD, Hall JC. Ischaemia-reperfusion injury to the intestine. Australian and New Zealand Journal of Surgery. 1998; 68(8): 554-561.

23) Kutala VK, Khan M, Angelos MG, Kuppusamy P. Role of oxygen in postischemic myocardial injury. Antioxidants and Redox Signaling. 2007; 9(8): 11931206.

24) LaVan FB, Hunt TK. Oxygen and wound healing. Clinics in Plastic Surgery. 1990; 17(3): 463-472.

25) Marti HJ, Bernaudin M, Bellail A, Schoch H, Euler M, Petit E, Risau W. Hypoxia-induced vascular endothelial growth factor expression precedes neovascularization after cerebral ischemia. The American Journal of Pathology. 2000; 156(3): 965-76.

26) Matsunami T, Sato $Y$, Hasegawa $Y$, Ariga S, Kashimura $\mathrm{H}$, Sato T, Yukawa M. Enhancement of reactive oxygen species and induction of apoptosis in streptozotocininduced diabetic rats under hyperbaric oxygen exposure. The International Journal of Clinical and Experimental Pathology. 2011; 4(3): 255-66.

27) Ngo AT, Riemann M, Holstein-Rathlou N, Pedersen CT, Jensen LJ. Significance of $\mathrm{K}_{\mathrm{ATP}}$ channels, L-type $\mathrm{Ca}^{2+}$ channels and CYP450-4A enzymes in oxygen sensing in mouse cremaster muscle arterioles. In vivo. BioMed Central Physiology. 2013; 13:8. 
28) Ngoh GA, Watson LJ, Facundo HT, Jones SP. Augmented O-GlcNAc signaling attenuates oxidative stress and calcium overload in cardiomyocytes. Amino Acids. 2011; 40(3): 895-911.

29) Nosál'ová V, Sotníková R, Drábiková K, Fialová S, Koštálová D, Banášová S, Navarová J.

Chemiluminescence response induced by mesenteric ischaemia/reperfusion: effect of antioxidative compounds ex vivo. Interdisciplinary Toxicology. 2010; 3(3): 105-108.

30) Park YY. Ischemia/reperfusion lung injury increases serum ferritin and heme oxygenase- 1 in rats. Korean Journal of Physiology and Pharmacology. 2009; 13(3): 191-197.

31) Perrelli MG, Pagliaro P, Penna C. Ischemia/reperfusion injury and cardioprotective mechanisms: Role of mitochondria and reactive oxygen species. World Journal of Cardiology. 2011; 3(6): 186-200.

32) Pohl U. Endothelial cells as part of a vascular oxygensensing system: hypoxia-induced release of autacoids. Experientia. 1990; 46(11-12): 1175-1179.

33) Quayle JM, Nelson MT, Standen NB. ATP-sensitive and inwardly rectifying potassium channels in smooth muscle. Physiological Review. 1997; 77(4): 1166-1232.
34) Rotstein OD. Pathogenesis of multiple organ dysfunction syndrome: gut origin, protection, and decontamination. Surgical Infection. 2000; 1(3): 217-225.

35) Siegel MP, Kruse SE, Knowels G, Salmon A, Beyer R, Xie H, Remmen HV, Smith SR, Marcinek DJ. Reduced coupling of oxidative phosphorylation in vivo precedes electron transport chain defects due to mild oxidative stress in mice. PLoS One. 2011; 6(11): e26963.

36) Springer TA. Traffic signals for lymphocyte recirculation and leukocyte emigration: the multistep paradigm. Cell. 1994; 76(2): 301-314.

37) Thom SR, Bhopale VM, Velazquez OC. Stem cell mobilization by hyperbaric oxygen. American Journal of Physiology. 2006; 290(4): 1378-1386.

38) Voet D, Pratt, CW Voet JG. (2011) Fundamentals of biochemistry: Life at the molecular level. Toronto: John Wiley \& Sons Canada, Limited. 4e.

39) Williams, S. The role of hyperbaric oxygen therapy in trauma. Trauma. 2010; 12(1): 13-20.

40) Zhang L, Bonev AD, Mawe GM, Nelson MT. Protein kinase A mediates activation of ATP-sensitive $\mathrm{K}^{+}$ currents by CGRP in gallbladder smooth muscle. American Journal of Physiology. 1994; 267(3): G494G499. 